\title{
Three years of AstroSat: Indian Astronomy mission
}

\author{
Girish Veerappa* \\ Space Science Program Office, ISRO Headquarters, Bangalore 5600231, INDIA
}

\begin{abstract}
The AstroSat is India's first multi-wavelength satellite dedicated for astronomy. It carries five scientific payloads covering a wide wavelength range (from visual/ultraviolet to high energy X-rays) and an auxiliary payload for monitoring in-situ charged particles. In this paper we describe the mission, the scientific payloads and a few important results from the AstroSat. The AstroSat is a proposal driven mission open for international users. The data is made public after a lock-in period of twelve months. We give a detailed description of the procedure to access archival data of the AstroSat. AstroSat has successfully completed three years in orbit and continues to yield valuable science data.
\end{abstract}

Keywords: Missions: AstroSat - Detectors: UV, X-rays

\section{Introduction}

The AstroSat is India's multi-wavelength astronomy mission (Agrawal 2006, Singh 2002) capable of obtaining simultaneous measurements in the energy bands in the ultraviolet (near and far) and $\mathrm{X}$-rays $(0.3 \mathrm{keV}$ to $100 \mathrm{keV})$. The AstroSat is a multi-institutional mission. Five of the six payloads onboard AstroSat are built by collaborating institutes. Four of them are co-aligned, namely the UltraViolet Imaging Telescope (UVIT), the Soft X-ray Telescope (SXT), the Large Area X-ray Proportional Counter (LAXPC), and the Cadmium Zinc Telluride (CZTI). The fifth payload, the Scanning Sky Monitor (SSM), is an all-sky monitor for transient detection and follow-up. The AstroSat also carries a sixth payload, the Charged Particle Monitor (CPM), for in-situ monitoring of high energy charged particles and to provide these measurements to the other payloads.

The AstroSat was launched into a low Earth orbit (LEO) of $650 \mathrm{~km}$ from the Sriharikota High Altitude Range (SHAR) on 28 September 2015 using the PSLV C30 XL rocket. A near equatorial orbit with an inclination of $\sim 6^{\circ}$ was chosen to reduce the effects of enhanced charged particle concentration over South Atlantic namely South Atlantic Anomaly (SAA). After injection into orbit, the two solar panels of AstroSat were automatically deployed in a quick sequence. The payloads were switched on sequentially starting with CPM, followed by CZTI, SSM, LAXPC, and SXT. The UVIT was the last payload to be turned on, almost two months after reaching the orbit, to avoid contamination due to out-gassing. The spacecraft control centre at the Mission Operations Complex (MOX) of ISRO Telemetry, Tracking and Command Network (ISTRAC), located in Bengaluru (India), manages the satellite over the entire life-time of the mission.

We briefly introduce the five scientific payloads onboard AstroSat followed by the current status and a few recent exciting results.

*girishv@isro.gov.in 


\section{Scientific Payloads}

Four payloads onboard AstroSat, i.e. UVIT, SXT, LAXPC, and CZTI, are co-aligned to enable simultaneous wide-band observations. This is a unique feature of AstroSat which is very useful for studying transient sources. Fig. 1 shows the energy coverage of the X-ray detectors of AstroSat. For comparison, the effective area and energy coverage of selected current and future X-ray observatories are also plotted.

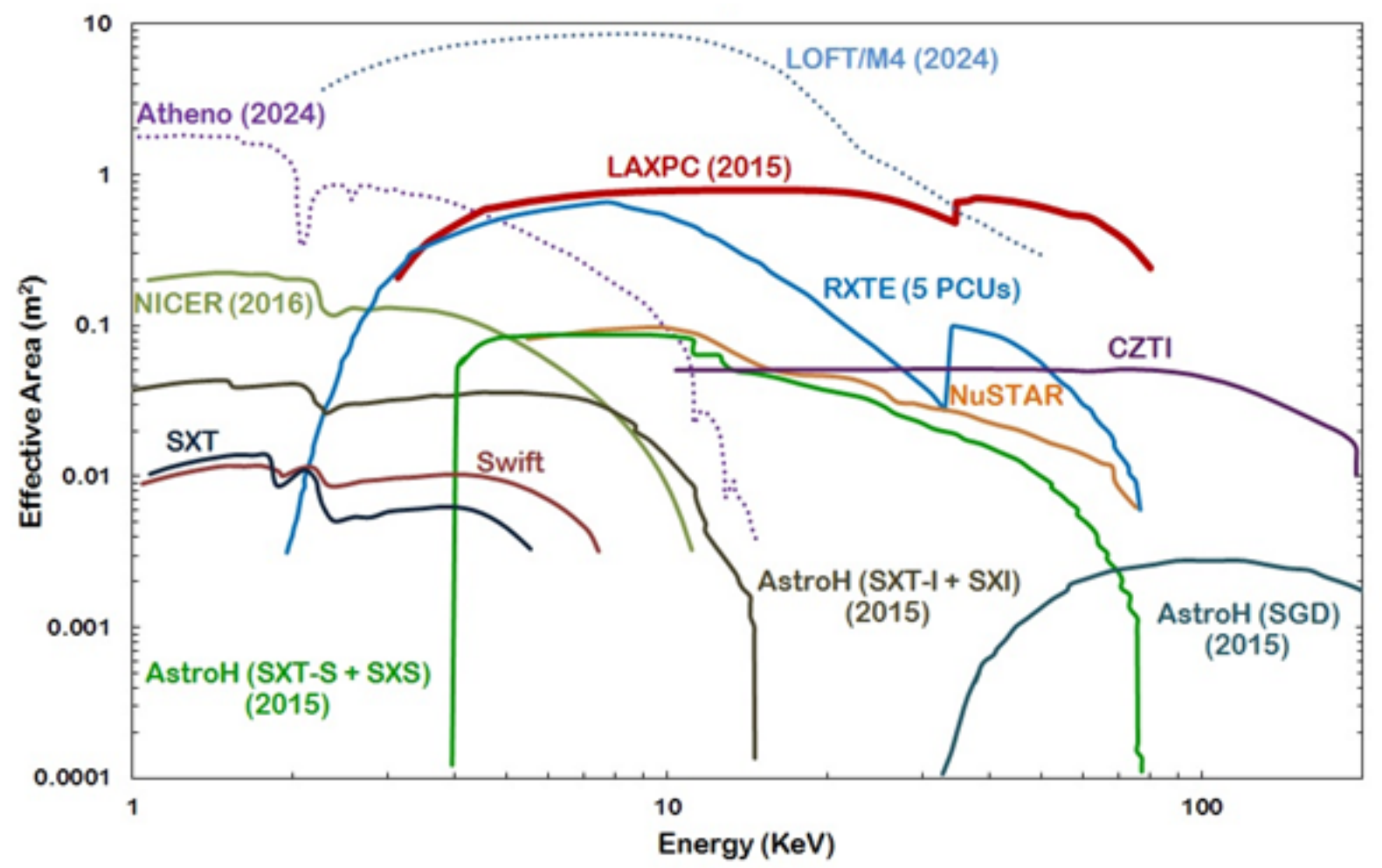

Figure 1: Energy coverage and the effective area of AstroSat X-ray payloads compared to the other observatories. Courtesy: LAXPC team, TIFR.

\subsection{Ultra-Violet Imaging Telescope (UVIT)}

The UVIT is designed and developed by the Indian Institute of Astrophysics (IIA; Bengaluru, India), in collaboration with the Inter University Centre for Astronomy and Astrophysics (IUCAA; Pune, India) and the Canadian Space Agency (CSA). The UVIT can simultaneously make observations in visible (VIS: $300-550 \mathrm{~nm}$ ), near ultraviolet (NUV: $200-300 \mathrm{~nm}$ ) and far ultraviolet (FUV: $130-180 \mathrm{~nm}$ ) via two similar Richey-Chretien telescopes with an aperture of $37.5 \mathrm{~cm}$ each (Fig. 2). The observations are made through narrow band filters. In one of the telescopes, the beam is split in two and fed to the VIS and NUV channels using a beam splitter. The UVIT uses three similar intensified CMOS sensors as detectors which are operated in photon counting mode.

The UVIT has a spatial resolution of $1.4-1.8^{\prime \prime}$ which is three times better than the Galaxy Evolution Explorer (GALEX; Girish et al. 2017). The maximum effective areas of the FUV and NUV are $12 \mathrm{~cm}^{2}$ and $50 \mathrm{~cm}^{2}$, respectively. The UVIT FUV has a sensitivity of 20 mag at $5 \sigma$ level for an integration of $200 \mathrm{~s}$. In comparison, the effective areas of the GALEX FUV and NUV are $36.8 \mathrm{~cm}^{2}$ and $61.7 \mathrm{~cm}^{2}$, respectively. The GALEX FUV has a sensitivity of $20 \mathrm{mag}$ at $5 \sigma$ level for an integration of $100 \mathrm{~s}$. For more information on the UVIT, readers are referred to Subramaniam (2012). 


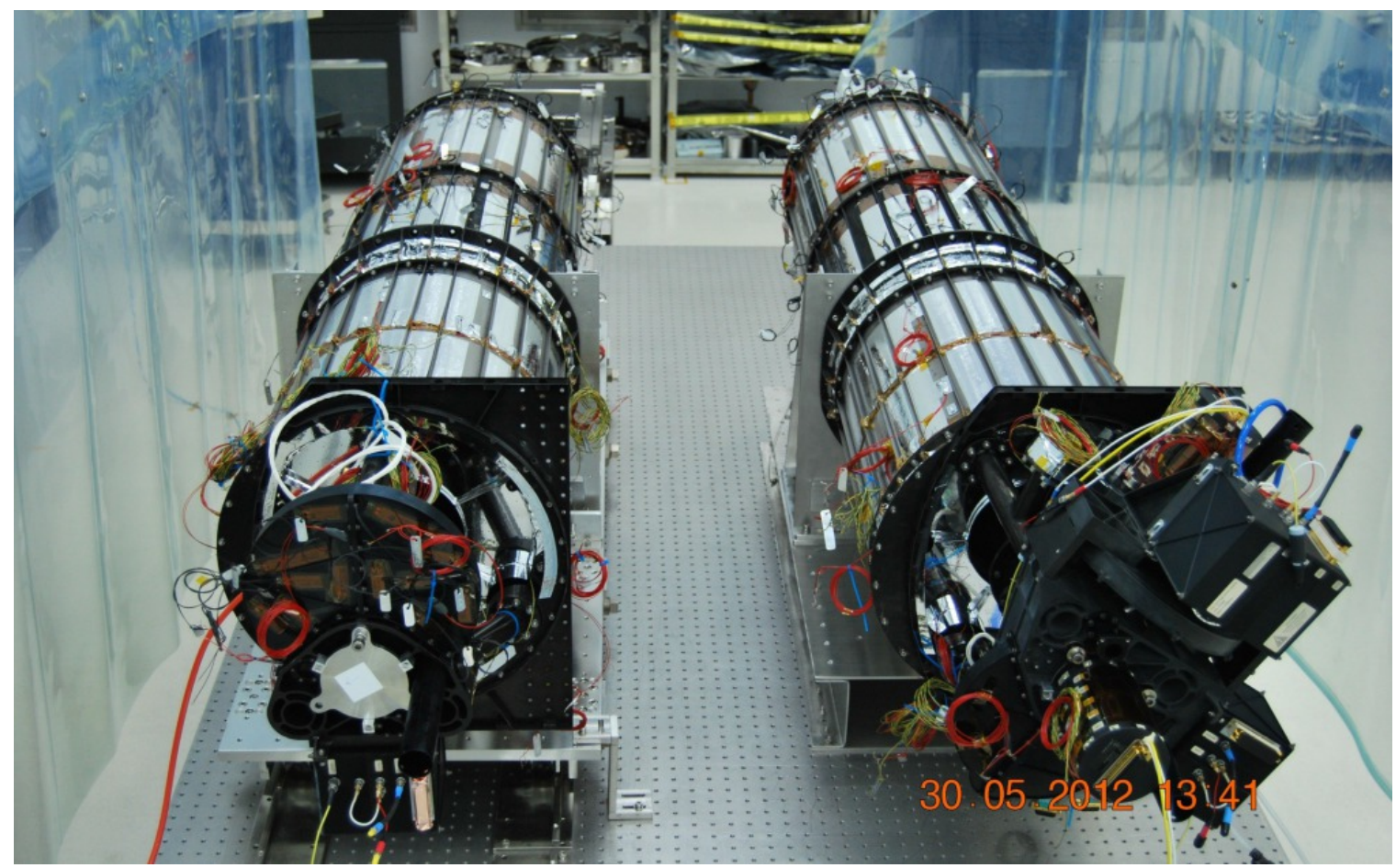

Figure 2: Twin telescopes of UVIT, feeding light to the FUV channel (left) and both the VIS and NUV channels (right).

\subsection{Soft X-ray Telescope (SXT)}

The SXT onboard AstroSat is developed by the Tata Institute of Fundamental Research (TIFR; Mumbai, India) in collaboration with the university of Leicester (United Kingdom). The SXT is an imaging telescope whose optics have a Wolter type I geometry made up of 320 gold coated aluminum foils nested together (Fig. 3). SXT operates in the energy range of 0.3 to $8.0 \mathrm{keV}$. The soft X-ray photons reflected from the gold foils are collected by a charge coupled device (CCD) camera cooled to $-80^{\circ} \mathrm{C}$ using active thermo-electric coolers (Fig. 3). The field of view (FOV) of the SXT is $40 \mathrm{arcmin}$. The geometric collecting area of the SXT is $250 \mathrm{~cm}^{2}$ providing a sensitivity of $15 \mu \mathrm{Crab}$ at $5 \sigma$ level for an integration of $10000 \mathrm{~s}$. The SXT has an energy resolution of $150 \mathrm{eV}$ at $6.0 \mathrm{keV}$ and a spatial resolution of 3 - 4 arcmin (Singh et al. 2016).
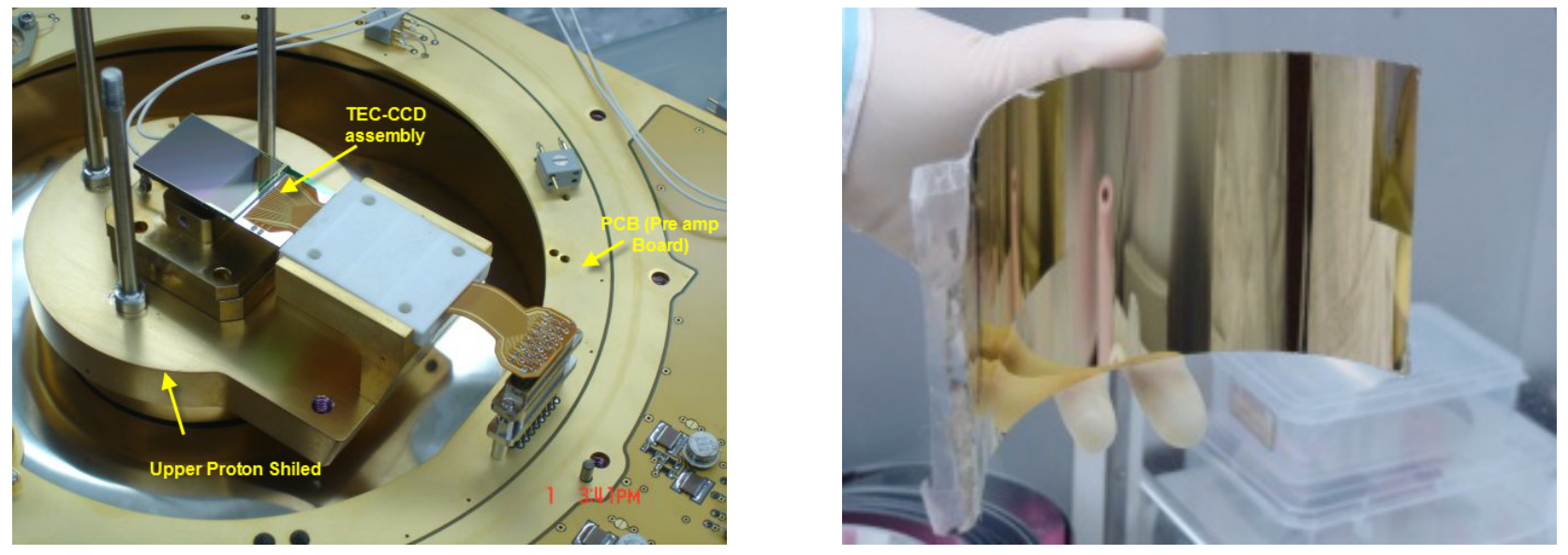

Figure 3: The SXT detector showing the CCD sensor (left), and one SXT gold plated mirror (right). 


\subsection{Large Area Proportional Counter (LAXPC)}

The LAXPC consists of three co-aligned and identical large area proportional counters filled with P10 (Xenon + Methane) gas maintained at a pressure of $\sim 2$ atmosphere. It operates in the energy range of $3-80 \mathrm{keV}$ with a moderate energy resolution of $11 \%$ at $22 \mathrm{keV}$. Due to its very large collecting area $\left(\sim 10800 \mathrm{~cm}^{2}\right)$, it can detect faint signals at high temporal resolution $(10 \mu \mathrm{s})$ and provide X-ray spectra at very short intervals (Yadav et al. 2016). The FOV of LAXPC is around $1^{\circ}$ determined by a mechanical collimator placed above each proportional counter. The LAXPC has a sensitivity of $0.1 \mathrm{mCrab}$ at $3 \sigma$ level for an integration of $500 \mathrm{~s}$. The LAXPC is developed by the TIFR.

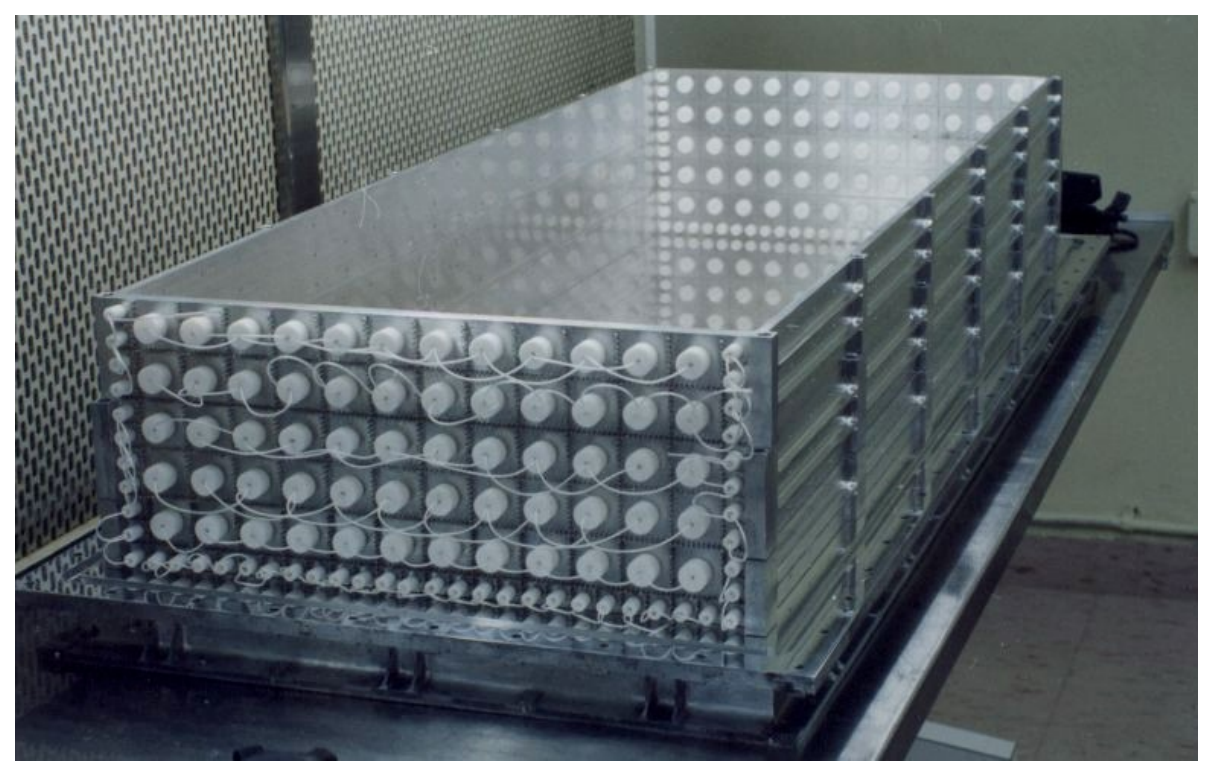

Figure 4: One of the three proportional counters of the LAXPC.

\subsection{Cadmium Zinc Telluride Imager (CZTI)}
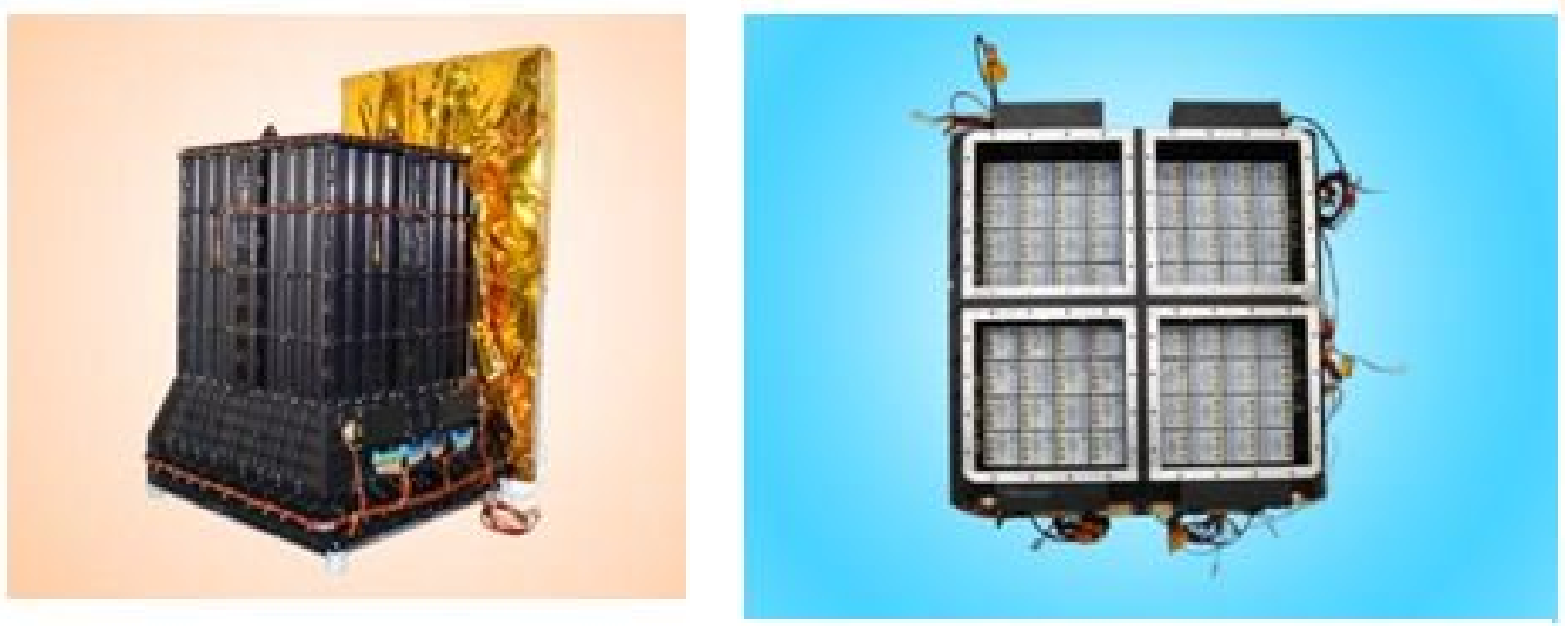

Figure 5: The CZTI payload onboard AstroSat (left) and one quadrant of the CZTI showing the pixellated CZT detectors (right). One module of CZTI consists of $16 \mathrm{CZT}$ detectors and each individual CZT detector has 256 pixels. 
The CZTI is a hard X-ray imaging instrument operating in the energy range of $10-100 \mathrm{keV}$ with a large collecting area of $\sim 1000 \mathrm{~cm}^{2}$. The sensors of the CZTI are solid state detectors, viz., pixellated CZT sensors. The detector has a detection efficiency of $95 \%$ in $10-100 \mathrm{keV}$ range.

A collimator made of tantalum defines the FOV of $4.6^{\circ} \times 4.6^{\circ}$ of the CZTI. A 2D coded aperture mask is used to obtain the imaging. Beyond $100 \mathrm{keV}$, the CZTI collimator does not stop the Xray photons, making the CZTI a gamma-ray burst (GRB) monitor. Fig. 5 shows the CZTI (left) and arrangement of the CZT sensors in the payload (right). The CZTI is developed by the TIFR in collaboration with the Vikram Sarabhai Space Centre (VSSC; Thiruvanthapuram, India) and the IUCAA. We refer to Rao et al. (2017) for more information on the CZTI. The CZTI team also developed the sixth payload (the CPM) onboard the AstroSat.

Though not designed for the X-ray polarization measurements, the pixelization of the detector has enabled the CZTI to be used as an X-ray polarization detector. The CZTI has measured polarization in several GRBs and detected for the first time X-ray polarization for the off-pulse region of the Crab pulsar (Vadawale et al. 2018).

\subsection{Scanning Sky Monitor (SSM)}

The SSM onboard AstroSat is the only scientific payload looking away from the co-aligned axis of the other payloads onboard the AstroSat. It consists of three position sensitive proportional counters mounted on a rotating platform (Fig. 6). These three cameras are combined with a one dimensional coded mask providing an angular resolution of $12^{\prime}$ along the coding direction and $2.5^{\circ}$ along the non-coding direction.
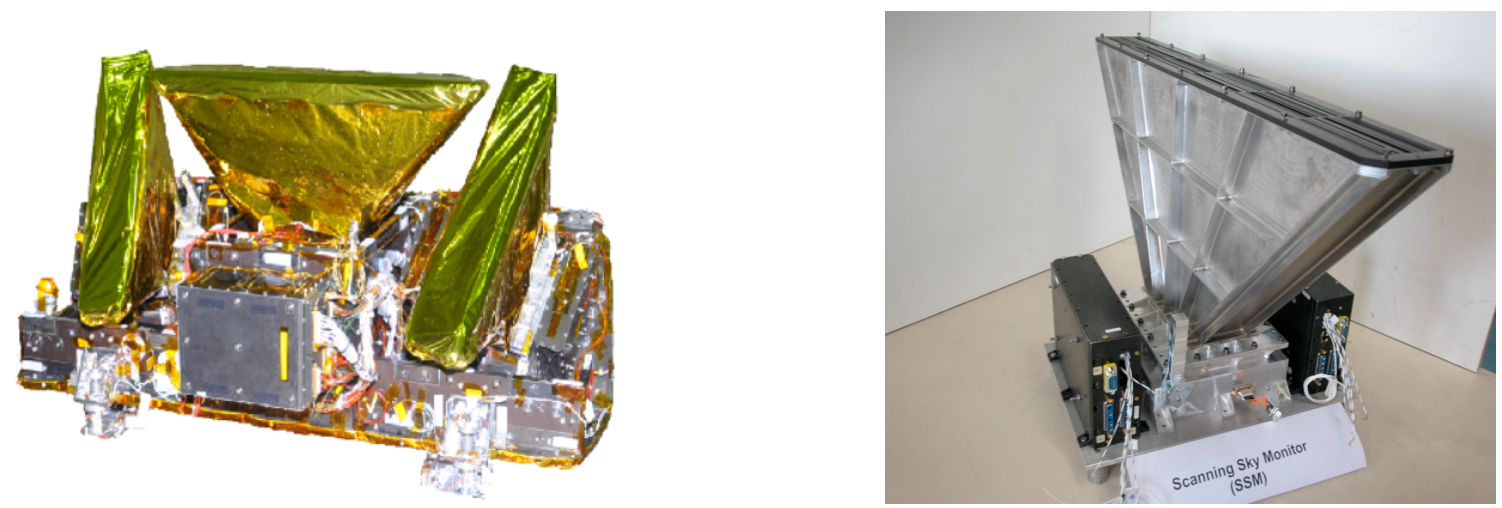

Figure 6: A total view of the AstroSat SSM mounted on the moving platform is given on the left while a single SSM detector with electronics boxes is shown on the right. The coded aperture mask is also seen at the top of the collimator.

The SSM is mounted on a rotating platform (Fig. 6, left) and operates in a step and stare mode with a step of $10^{\circ}$ and a stare time of 10 minutes in each step. The FOV of the central SSM (SSM3) is $22^{\circ} \times 100^{\circ}$ while the FOV of the two edge SSM cameras (SSM1 and SSM2) is $\sim 26.8^{\circ} \times 100^{\circ}$. The two edge cameras are slanted to provide an overlapping region close to the center of their FOV, as shown in Fig. 7. This slanted arrangement of the SSM cameras enables a better localization along the long axis of the FOV.

The sky coverage of the SSM in one full rotation is shown on the right-hand side of Fig. 7 which is close to $50 \%$ of the sky. The SSM operates in the energy range of $3-12 \mathrm{keV}$ with a coarse energy resolution of about $25 \%$ at $6 \mathrm{keV}$. The geometric area of the SSM is $\sim 58 \mathrm{~cm}^{2}$ per SSM camera providing a sensitivity of $\sim 30 \mathrm{mCrab}$ at $3 \sigma$ level for an integration of $600 \mathrm{~s}$. Compared to the SSM, the All Sky Monitor (ASM) onboard the Rossi X-ray Timing Explorer (RXTE) has three cameras with a FOV of $6^{\circ} \times 90^{\circ}$ and a sensitiviy close to twice the sensitivity of the SSM cameras. 

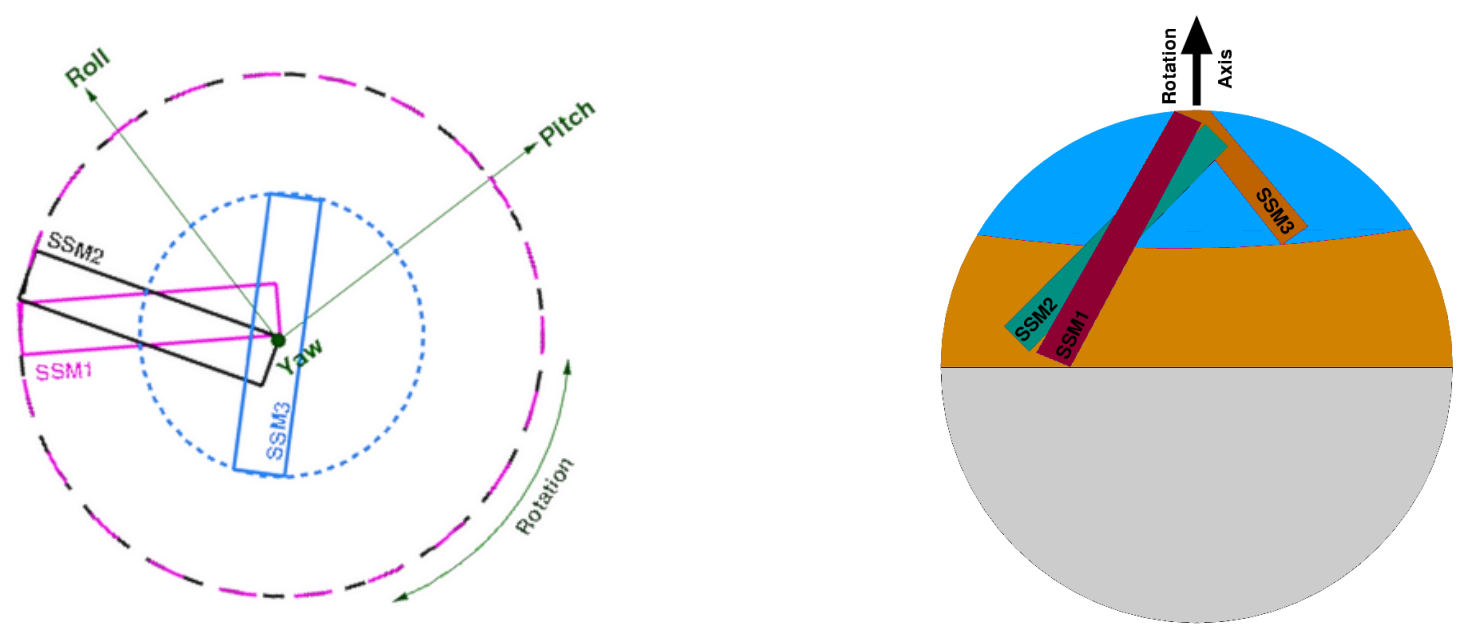

Figure 7: The field of view (left) and sky coverage (right) of the three SSM cameras. Courtesy: SSM team, URSC.

The SSM is developed by the U R Rao Satellite Center (URSC; Bengaluru, India; formerly known as the ISRO Satellite Centre) in collaboration with the IUCAA (Seetha et al. 2006).

\section{AstroSat Usage}

As mentioned earlier, the AstroSat was launched in September 2015. The first six months after the launch, AstroSat observations were made for performance and verification (PV) purposes. This PV phase was followed by a twelve month guaranteed time for the payload teams. The AstroSat works as a proposal based observatory. The call for proposals for observations are issued usually in February/March of each year for an observation cycle starting from October of that year.

To support the user community, the ISRO has set-up the AstroSat Support Cell (ASC) at the IUCAA. In addition to the development of a new database of active AstroSat users, the ASC also supports the user community from the proposal submission stage to data analysis. The readers can reach the ASC through http://astrosat-ssc.iucaa.in or by email on "astrosathelp@iucaa.in."

After a lock-in period of twelve months, the AstroSat observations are made available to the public as archival data hosted on https://astrobrowse.issdc.gov.in maintained by the ISRO Space Science Data Centre (ISSDC; Bengaluru, India) and the ISRO Telemetry and Tracking Centre (ISTRAC; Bengaluru, India). Anybody can download data through this website after registering with their email. ISSDC hosts data of both level 1 (calibrated data) and level 2 (scientific data). The level 2 data is produced using standard filters and calibration data.

The AstroSat calibration database (CALDB) is hosted by the AstroSat support cell (http://astrosatssc.iucaa.in/) along with the software required for analysing the AstroSat data (pipeline software and level 2 software) and recipes. The level 2 data generated using the AstroSat pipeline software is in the standard FITS format and compatible with popular analysis tools like IRAF, HEASOFT, XSPEC, etc. The AstroSat pipeline and level 2 software runs on Linux/Unix platforms.

As on January 2019, there were 973 registered users of AstroSat from 43 countries around the globe. About half of them originate from India. Fig. 8 shows the distribution of these users. For clarity, only the countries with more than ten users are shown in the figure. All the others grouped together. For the next cycle starting from 1 October 2019, the share of AstroSat observation time reserved for international researchers will be $28 \%$, including the share of CSA and the university of Leicester. 


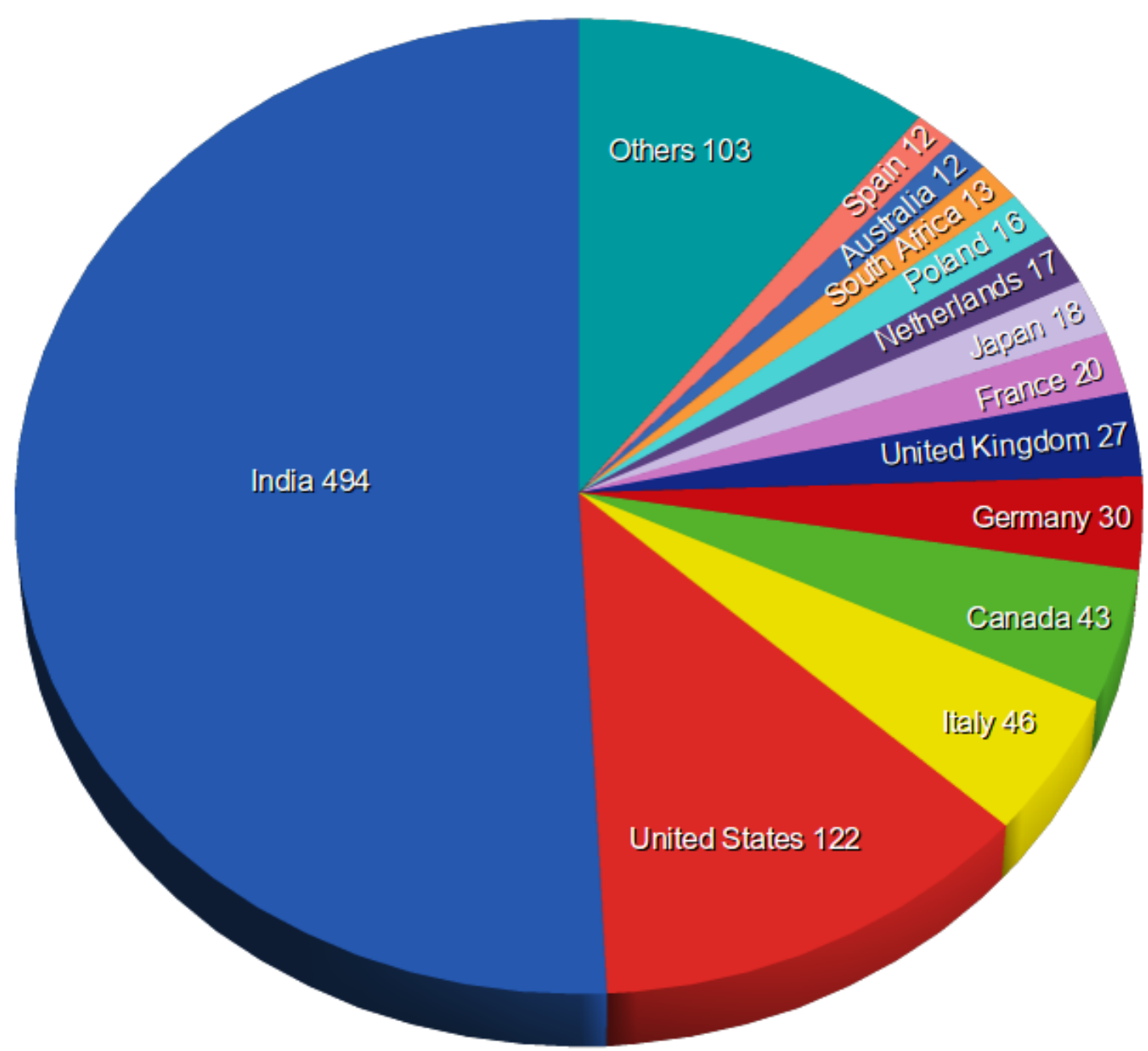

Figure 8: Distribution of registered AstroSat users from around the globe. The countries with at maximum ten users are grouped together for clarity.

\section{Results from AstroSat}

The AstroSat has produced many exciting new results and is expected to continue its operations for another two years. In January 2019, more than 250 papers based on AstroSat data were already published including 100 in national and international refereed journals. This number is expected to grow steadily because the data has become open to the public at the end of September 2018.

Some major recent results from AstroSat are

1. CZTI Observations of GRB 151006: Demonstration of polarization measurement capability of the CZTI using the compton effect (Rao et al. 2016)

2. Detection of the polarization of the Crab pulsar (Vadawale et al. 2018)

3. Detection of a rare triple burst in a low-mass X-ray binary (Beri et al. 2018)

4. Discovery of an extended FUV emission from the Butterfly nebular NGC 6302 (Kameshwara et al. 2018)

5. Violation of the synchrotron line of death by the highly polarized GRB 160802A (Chand et al. 2018) 
The AstroSat is designed to operate for five years and is expected to remain operational for a longer time. The ISRO has assured to keep AstroSat in operation as long as it keeps delivering scientific data.

\section{Acknowledgements}

The authors would like to thank the Department of Science and Technology (DST), India for the travel support to attend the BINA meeting under Indo-Belgium "BINA" projects grant number DST/INT/Belg/P02 and the Royal Observatory of Belgium for the hospitality during the meeting under the grant BL/11/IN07.

\section{References}

Agrawal P. C. 2006, AdSpR, 38, 12

Beri A., Paul B., Yadav J. S. 2018, MNRAS, 482, 4, 4397

Chand V., Chattopadhyay T., Iyaani S. et al. 2018, ApJ, 862, 154

Girish V., Tandon S. N., Sriram N. et al. 2017, ExA, 43, 59

Kameswara Rao N., De Marco O., Krishna S. et al. 2018, A\&A, 620, 138

Rao A. R., Bhattacharya D., Bhalerao V. B. et al. 2017, CurSci. 113, 595

Rao A. R., Chand V., Hingar M. K. et al. 2016, ApJ, 833, 86

Seetha S., Ramadevi M. C., Babu V. C. et al. 2006, AdSpR, 38, 12, 2995

Singh K. P., AstroSat team 2002, BASI, 30, 803

Singh K. P., Stewart G. C., Chandra S. et al. 2016, SPIE, 9905, 10

Subramaniam A. 2012, Astronomical Society of India Conference Series, 4, 165

Vadawale S. V., Chattopadhyay T., Mithun, N. P. S. 2018, NatAs, 2, 50

Yadav J. S., Agrawal P. C., Antia H. M. et al. 2016, SPIE, 9905, 15 